\title{
Assessment of ventricular flow dynamics by 4D-flow MRI in patients following surgical repair of $d$-transposition of the great arteries
}

\author{
Fraser M. Callaghan ${ }^{1,2,3}$ (D) Barbara Burkhardt ${ }^{3,4}$ - Emanuela R. Valsangiacomo Buechel ${ }^{3,4}$. \\ Christian J. Kellenberger ${ }^{3,5} \cdot$ Julia Geiger ${ }^{3,5}$
}

Received: 26 November 2020 / Revised: 18 January 2021 / Accepted: 18 February 2021 / Published online: 30 March 2021

(C) The Author(s) 2021

\begin{abstract}
Objectives To use 4D-flow MRI to describe systemic and non-systemic ventricular flow organisation and energy loss in patients with repaired d-transposition of the great arteries (d-TGA) and normal subjects.

Methods Pathline tracking of ventricular volumes was performed using 4D-flow MRI data from a 1.5-T GE Discovery MR450 scanner. D-TGA patients following arterial switch $(n=17$, mean age $14 \pm 5$ years $)$ and atrial switch $(n=15,35 \pm 6$ years $)$ procedures were examined and compared with subjects with normal cardiac anatomy and ventricular function $(n=12,12 \pm 3$ years). Pathlines were classified by their passage through the ventricles as direct flow, retained inflow, delayed ejection flow, and residual volume and visually and quantitatively assessed. Additionally, viscous energy losses $\left(\mathrm{EL}_{v}\right)$ were calculated.

Results In normal subjects, the ventricular flow paths were well ordered following similar trajectories through the ventricles with very little mixing of flow components. The flow paths in all atrial and some arterial switch patients were more irregular with high mixing. Direct flow and delayed ejection flow were decreased in atrial switch patients' systemic ventricles with a corresponding increase in residual volume compared with normal subjects ( $p=0.003$ and $p<0.001$ respectively) and arterial switch patients $(p<$ 0.0001 and $p<0.001$ respectively). In non-systemic ventricles, arterial switch patients had increased direct flow and decreased delayed ejection fractions compared to normal $(p=0.007$ and $p<0.001$ respectively) and atrial switch patients $(p=0.01$ and $p<$ 0.001 respectively). Regions of high levels of mixing of ventricular flow components showed elevated $\mathrm{EL}_{v}$.

Conclusions 4D-flow MRI pathline tracking reveals disordered ventricular flow patterns and associated $\mathrm{EL}_{v}$ in d-TGA patients. Key Points

- 4D-flow MRI can be used to assess intraventricular flow dynamics in d-TGA patients.

- $d$-TGA arterial switch patients mostly show intraventricular flow dynamics representative of normal subjects, while atrial switch patients show increased flow disorder and different proportions of intraventricular flow volumes.

- Flow disruption and disorder increase viscous energy losses.
\end{abstract}

Keywords Transposition of great vessels $\cdot$ Ventricular function $\cdot$ Magnetic resonance imaging $\cdot$ Viscosity

Fraser M. Callaghan

fraser.callaghan@kispi.uzh.ch

1 University of Zurich, Zurich, Switzerland

2 Center for MR-Research, University Children's Hospital, Steinwiesstrasse 75, 8032 Zurich, Switzerland

3 Children's Research Center, University Children's Hospital, Zurich, Switzerland

4 Division of Pediatric Cardiology, University Children's Hospital, Zurich, Switzerland

5 Department of Diagnostic Imaging, University Children's Hospital, Zurich, Switzerland

$\begin{array}{ll}\text { Abbreviations } & \\ \overline{E L_{v}} & \text { Normalised viscous energy loss (by EDV) } \\ \text { 4D-flow MRI } & \text { Four-dimensional flow MRI } \\ \text { BSA } & \text { Body surface area } \\ \text { d-TGA } & \text { d-Transposition of the great arteries } \\ \text { EDV } & \text { End diastolic volume } \\ \text { EF } & \text { Ejection fraction } \\ \text { EL }_{v} & \text { Viscous energy loss } \\ \text { MV } & \text { Mitral valve } \\ \text { SV } & \text { Stroke volume } \\ \text { TV } & \text { Tricuspid valve }\end{array}$




\section{Introduction}

Dextro-transposition of the great arteries (d-TGA) is a congenital heart defect characterised by the transposition of the primary arteries exiting the heart resulting in deoxygenated blood being passed to the aorta and returned to the body, while oxygenated blood is returned from the left ventricle to the lungs in a closed circuit. D-TGA accounts for $5-7 \%$ of all congenital heart defects and requires surgical intervention to ensure patient survival [1]. Previously, patients have been treated with an atrial switch procedure, redirecting pulmonary blood from the lungs to the right ventricle and thus to the transposed aorta and body; however, this has been gradually replaced by the arterial switch procedure, which transects the aorta and main pulmonary artery, returning them to their natural position and resulting in improved survival rates [2]. With arterial switch operations only surpassing atrial switch corrections in the early 1990s [2], the ongoing evaluation and assessment of surgically corrected d-TGA patients is mandatory to assess mid- and long-term sequelae.

Whereas short-term follow-up is excellent in most of the patients, there are typical potential mid- to long-term complications for both operations [3-6]. Atrial switch patients, after a Senning procedure, face a higher risk of atrial or ventricular arrhythmia as the right ventricle must maintain systemic circulation [3, 4]. This patient cohort also requires regular follow-up imaging by echocardiography and MRI to recognise pathologies that need re-interventions, mostly concerning the right ventricular outflow tract $[1,2,7,8]$.

4D-flow MRI provides a time-dependent, high-resolution velocity vector field, able to be interrogated retrospectively for a number of features such as flow volumes, flow patterns, pressure differences, energy loss, and wall shear stress [9, 10]. The technique is showing valuable promise in the area of congenital heart disease [11] and previous studies using flow-derived parameters such as kinetic energy and viscous energy loss $\left(\mathrm{EL}_{v}\right)$ in patients with tetralogy of Fallot or Fontan palliation have shown the potential of 4D-flow MRI for a more comprehensive intraventricular assessment compared to traditional volumetrics [12-15].

4D-flow MRI data in healthy subjects has shown that there are well-defined flow fractions that pass through the ventricles relative to the cardiac cycle [16-18]. These comprise a direct flow fraction, retained inflow, delayed ejection, and residual flow volume. By using this approach, we aim to assess the systemic and non-systemic ventricular dynamics of d-TGA patients following either atrial or arterial switch repair as well as normal subjects. We predict that the flow dynamics in dTGA subjects will be disturbed and deviate from that of normal subjects which in turn will have implications on intraventricular energetics.

\section{Methods}

\section{Patients}

Patients presenting for routine cardiac MRI assessment between April 2018 and October 2019 were retrospectively included in this study if they provided written, informed consent for retrospective use of their imaging data and if they had a corrected d-TGA (atrial switch repair according to Senning or arterial switch repair) or were found to have normal cardiac function and anatomy following examination.

\section{Data acquisition}

All subjects underwent cardiac MRI examination as part of their clinical assessment. All scanning was performed on a 1.5-T GE Discovery MR450 scanner using a 32 channel cardiac coil. As part of a standard clinical examination, shortaxis, four-chamber, two-chamber, and ventricle outflow tract CINE MRI views were obtained, as well as a 4-point 4D-flow acquisition covering the entire chest extending from below the heart inferiorly, to above the origin of the aortic arch branches superiorly. 4D-flow sequences were electrocardiogram gated with free breathing and were performed following a contrastenhanced agent (Dotarem $\AA$; gadoterate meglumine) magnetic resonance angiography sequence (mean time between contrast injection and 4D-flow sequence was $4.4 \pm 3.9 \mathrm{~min}$ ). Acquisitions had axial slice orientation, echo time was $2.4 \pm$ $0.04 \mathrm{~ms}$, repetition time $4.3 \pm 0.1 \mathrm{~ms}$, and a flip angle of $15^{\circ}$ was used. Velocity encoding ranged from 160 to $200 \mathrm{~cm} / \mathrm{s}$ dependent on initial 2D phase-contrast acquisitions at the ascending aorta and main pulmonary artery. Spatial resolution was acquired at a range of $1.3-2.7 \mathrm{~mm}$ in-plane resolution (median $2.0 \mathrm{~mm}$ ), reconstructed to $0.9-1.6 \mathrm{~mm}$ (median 1.4) and slice thickness ranged from 1.8 to $2.7 \mathrm{~mm}$ (median 2.0 $\mathrm{mm}$ ). The mean temporal resolution was $33.9 \pm 9.2 \mathrm{~ms}$ equating to 20-25 phases per cardiac cycle. A local shim volume covering the heart and major vessels was defined, segmentation factor of three was used and a $\mathrm{k}-\mathrm{t}$ acceleration parallel imaging technique $(k a t-A R C=8)$ was employed [19].

\section{Data analysis-volumetry}

Using four-chamber, two-chamber, and outflow tract views for correct orientation, ventricle lumen contours were drawn at end-diastolic and end-systolic time points on the short-axis images for both ventricles using a semi-automated technique in the Medis QMass software (version 8.1). The resulting enddiastolic volume (EDV), stroke volume (SV), and ejection fraction (EF) were calculated. Unless otherwise specified, EDV and SV are reported indexed by body surface area (BSA). 


\section{Data analysis-4D-flow post processing}

4D-flow MRI data were inspected manually for phase aliasing and data quality (an absence of patient movement or significant wrap around or ghosting artefact) and were corrected for background phase offsets using a $4^{\text {th }}$-order polynomial fit to static tissue. Mitral (MV) and tricuspid valve (TV) annulus planes were marked and tracked over time manually using four-chamber CINE scans. At each 4D-flow acquisition time step, the valve orifice was manually drawn on a reformatted 4D-flow image at the level of the corresponding MV and TV annulus plane. At the aortic and pulmonary valves, ROIs were manually drawn tracking the artery movements over time using just the 4D-flow data. To avoid confusion, ventricles are classified as systemic or non-systemic ventricles, depending on the circulation that they supply.

Pathline tracking was performed using the scientific visualisation software ParaView (Kitware Inc.). Source points were defined over an isotropic domain covering the end-diastolic ventricular volume but not crossing the inlet or outlet ROIs. The particle points were tracked both forwards and backwards over time starting from end-diastole, for one cardiac cycle using a $4^{\text {th }}$-order Runge-Kutta integration scheme. Particles that passed through the inlet ROI were considered to have originated from the left atrium or pulmonary veins (in the case of the systemic ventricle), while particles that crossed the outlet ROI were considered to be ejected from the ventricle during systole. Thus, particles that originated from the same source point and passed through the inlet ROI during backward time tracking and passed through the outlet ROI during forward time tracking were classified as direct flow according to the definitions of Eriksson et al [16]. Similarly, retained inflow-those entering the ventricle during diastole but not being ejected during the following systolic phase; delayed ejection-those residing within the ventricle at end-diastole and exiting during ejection; and residual volume - those residing in the ventricle at enddiastole and not being assigned to one of the other classifications were also identified. The accuracy of the flow particle classification was evaluated by comparing ventricle inflow and outflow volumes and by comparing the ejected volume independently against the stroke volume calculated from the short-axis volumetry. The path of travel of particles was assessed visually with respect to their classifications.

In addition, total $\mathrm{EL}_{v}$ was calculated over the cardiac cycle. $\mathrm{EL}_{v}$ are irreversible losses to thermal energy resulting from the frictional (viscous) interaction of fluid flow with itself and its boundaries. The instantaneous rate of $\mathrm{EL}_{v}$ can be calculated from the 3D velocity field $(\nu)$ as:

$\phi_{v}=\frac{1}{2} \sum_{i} \sum_{j}\left[\left(\frac{\partial v_{i}}{\partial x_{j}}+\frac{\partial v_{j}}{\partial x_{i}}\right)-\frac{2}{3}(\nabla \cdot v) \partial_{i j}\right]^{2}$ where $i$ and $j$ describe principal directions in the domain $x$ and $\partial_{i j}$ is the Kronecker delta. The rate of energy loss within the ventricle is the product of blood viscosity, assumed constant at $3.5 \times 10^{-3} \mathrm{~Pa} \cdot \mathrm{s}$, and the summation of $\phi_{v}$ over the ventricle volume. Total energy loss, measured in Joules, over a cardiac cycle is calculated by the integration of the rate of energy loss over time [20]. Given the variability in the study cohort, a normalised, by end-diastolic volume, total viscous energy loss $\left(\overline{E L_{v}}\right)$ was also calculated.

\section{Statistical analysis}

Data analysis was performed using the public environment for statistical computing R (version 3.2.3). Unless specified, data are expressed as mean \pm one standard deviation. Data for each flow classification component (direct flow, delayed ejection, and retained inflow) and each ventricle (systemic or non-systemic) were tested for normality via the Shapiro-Wilk test and compared with one-way ANOVA and then between arterial and atrial switch dTGA groups and normal subjects using Student's $t$ test. Significance is defined at a level of $p<0.05$.

\section{Results}

\section{Study cohort and ventricle volumetrics}

The study included $15 \mathrm{~d}$-TGA patients following atrial switch repair, $17 \mathrm{~d}$-TGA patients after arterial switch procedure, and 12 patients with normal cardiovascular anatomy and function. One subject from each cohort group was rejected for poor quality short-axis cine data that prevented ventricle segmentation. One additional arterial switch subject was excluded due to excessive aortic insufficiency. All included subjects had good-quality 4D-flow MRI data and quality control measures confirmed pathline volume fractions to within $13 \%$ agreement with ventricular stroke volumes. Full demographic and ventricle volumetric details are provided in Table 1 and illustrated in Fig. 1. Importantly, atrial switch patients were significantly older and had higher BSA values $(p<0.001$ for both) than both arterial switch and normal anatomy patients, whereas age and BSA were similar between normal and arterial switch patients. Atrial switch subjects had significantly larger, normalised, systemic ventricle EDV and lower systemic ventricle EF compared with patients after arterial switch procedure and those with normal anatomy. In the non-systemic ventricle, EF was slightly higher in both atrial and arterial switch patients compared to normal patients $(p=0.007$ and 0.039 respectively). 
Table 1 Demographics and normalised ventricular volume measurements

$p$ values

Atrial switch Arterial switch Normal Atrial vs arterial switch Normal vs atrial switch Normal vs arterial switch

\begin{tabular}{|c|c|c|c|c|c|c|}
\hline$N$ & 15 & 17 & 12 & & & \\
\hline Gender M/F (\%M) & $10 / 5(67 \%)$ & $11 / 6(65 \%)$ & $8 / 4(67 \%)$ & & & \\
\hline Age $[\mathrm{yr}]$ & $35 \pm 6$ & $14 \pm 5$ & $12 \pm 3$ & $<0.0001$ & $<0.0001$ & 0.18 \\
\hline $\mathrm{BSA}\left[\mathrm{m}^{2}\right]$ & $1.82 \pm 0.16$ & $1.41 \pm 0.4$ & $1.42 \pm 0.3$ & 0.001 & 0.0006 & 0.99 \\
\hline \multicolumn{7}{|l|}{ Volumetrics } \\
\hline \multicolumn{7}{|l|}{ Systemic ventricle } \\
\hline $\mathrm{EDV}\left[\mathrm{mL} / \mathrm{m}^{2}\right]$ & $112.7 \pm 27.6$ & $89.3 \pm 13.9$ & $76.2 \pm 14.9$ & 0.004 & 0.0003 & 0.02 \\
\hline $\mathrm{SV}\left[\mathrm{mL} / \mathrm{m}^{2}\right]$ & $48.0 \pm 13.2$ & $51.6 \pm 9.5$ & $45.2 \pm 10.3$ & 0.38 & 0.59 & 0.12 \\
\hline $\mathrm{EF}[\%]$ & $42.9 \pm 7.4$ & $57.9 \pm 5.8$ & $59.1 \pm 4.7$ & $<0.0001$ & $<0.0001$ & 0.31 \\
\hline \multicolumn{7}{|c|}{ Non-systemic ventricle } \\
\hline $\mathrm{EDV}\left[\mathrm{mL} / \mathrm{m}^{2}\right]$ & $76.2 \pm 20.9$ & $85.8 \pm 13.0$ & $82.9 \pm 18.6$ & 0.13 & 0.60 & 0.31 \\
\hline $\mathrm{SV}\left[\mathrm{mL} / \mathrm{m}^{2}\right]$ & $47.5 \pm 14.8$ & $50.5 \pm 7.2$ & $44.4 \pm 10.6$ & 0.46 & 0.52 & 0.069 \\
\hline $\mathrm{EF}[\%]$ & $62.0 \pm 6.6$ & $59.1 \pm 4.6$ & $53.8 \pm 6.9$ & 0.16 & 0.007 & 0.039 \\
\hline
\end{tabular}

\section{Visual analysis of ventricle flow dynamics}

Visually, the flow dynamics followed an ordered and distinctly structured profile for all normal subjects. In the systemic ventricle, the direct flow and retained inflow portions entered and moved towards the apex, inducing circulation in a path from the mitral valve towards the apex and then following the septal wall towards the outflow tract, driving the ventricle volumes already present at end-systole (delayed ejection and residual volume) in this direction. The direct flow portion was more centrally orientated and continued following this path to be ejected during the following systolic phase. The retained inflow portion moved along the ventricle free-wall and was retained in the ventricle during the following systolic phase. The delayed ejection portion filled the outflow
Fig. 1 Box and whisker plots showing the distribution of (BSA normalised) end-diastolic volume, stroke volume, and ejection fraction in the systemic (left column), and non-systemic (right column) ventricles of dTGA atrial switch and arterial switch patients and normal subjects. Boxes depict median (middle bar), upper and lower quartile values. Outliers are data $1.5 \mathrm{x}$ the inter-quartile range and are indicated with dots.

Significant differences between cohort measures are indicated by $* p<0.05 ; * * p<0.01 ; * * * p<$ 0.001

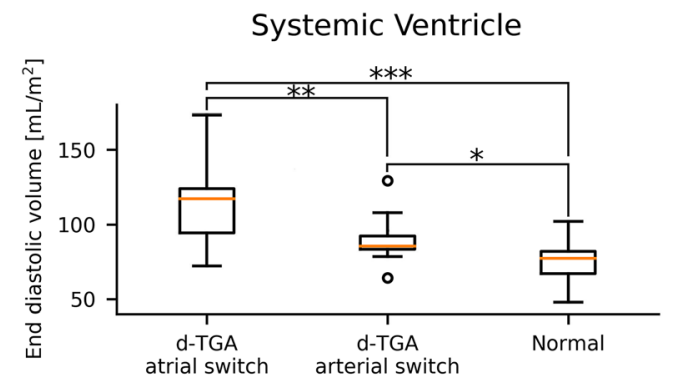

Non-systemic Ventricle
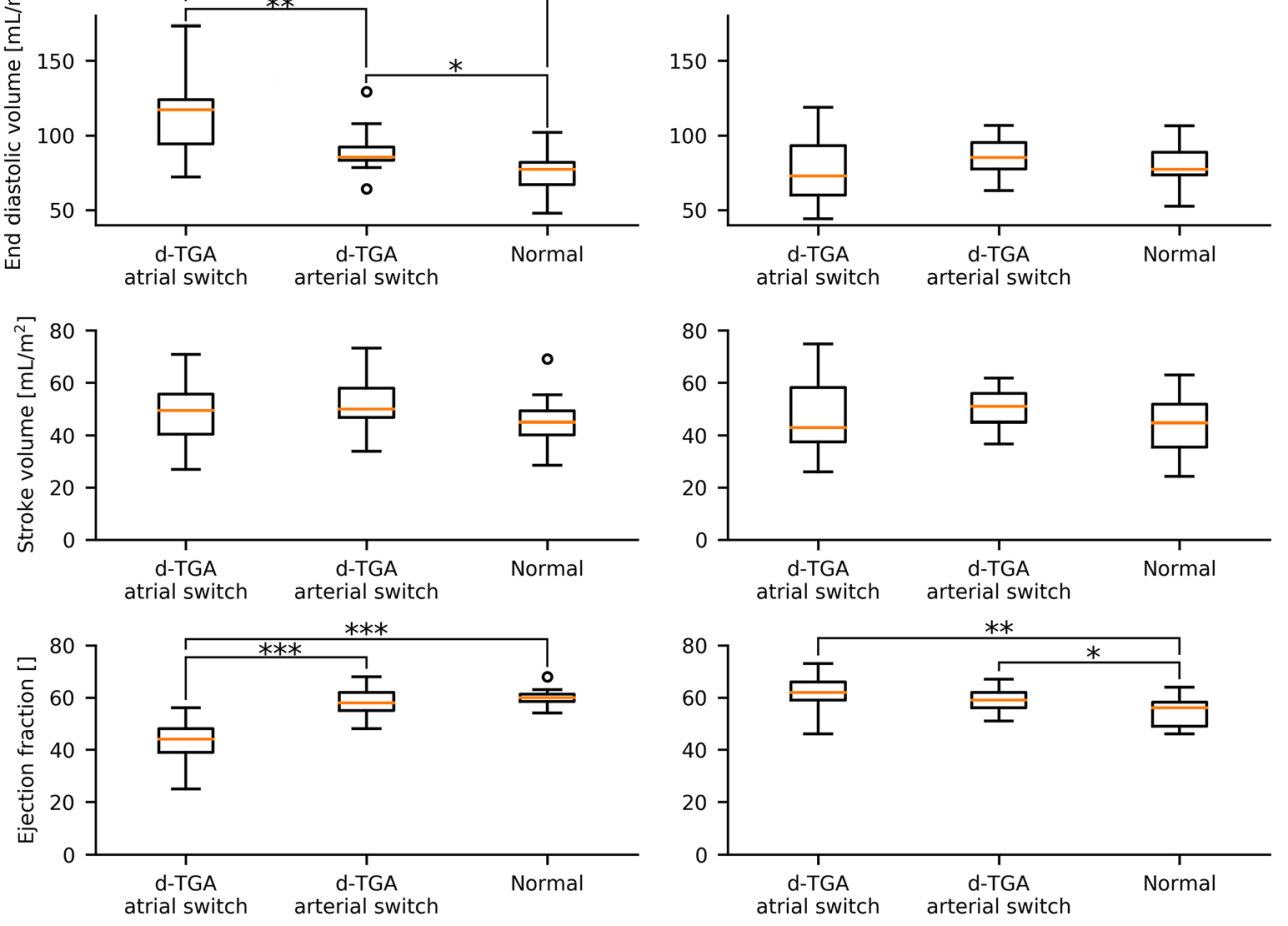
tract and posterior-septal wall region of the ventricle at end-diastole and was ejected during systole. Figure 2a and $b$ show the distribution of flow portions at enddiastole for a normal subject.

In the non-systemic ventricle, the retained inflow appeared to diverge into the various trabeculations which had been compressed at end-systole and the direct flow portion made a very direct path from tricuspid valve to pulmonary valve (Fig. 3a and b).
In arterial switch patients, the flow patterns in the systemic and non-systemic ventricles typically mimicked that of the normal subjects. In subjects who showed dissimilar flow patterns, increased mixing between the direct flow and delayed ejection components was obvious at end-diastole, which were then ejected together over systole (Figs. $2 c, d$ and $3 c, d$ ).

Atrial switch patients showed variable flow dynamics which deviated from those observed in both the systemic ventricles and non-systemic ventricles of the normal
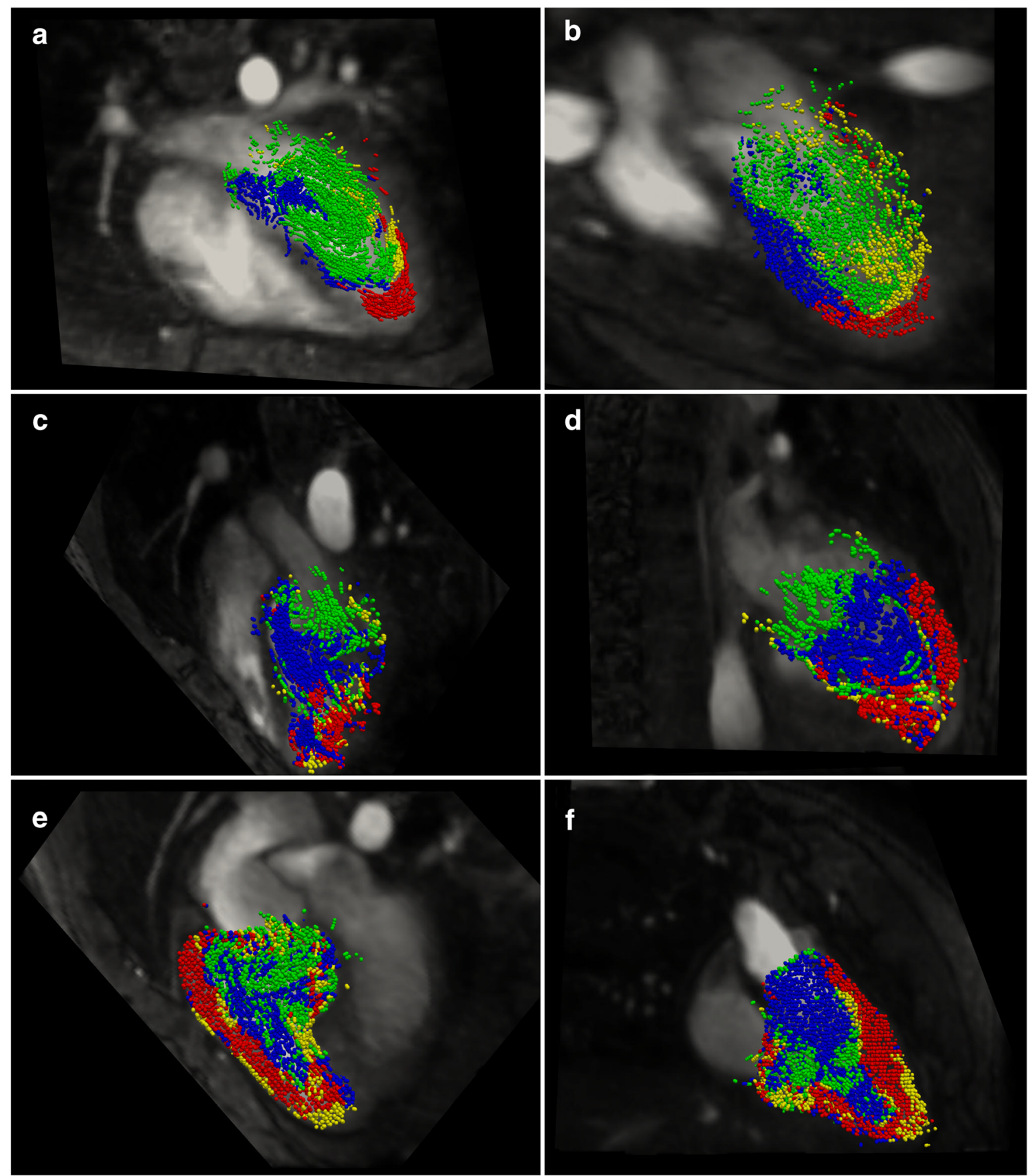

Fig. 2 The location of systemic ventricular blood volumes at the time point of end-diastole on two orthogonal views in a representative normal (a and b), d-TGA arterial switch (c and d), and a d-TGA atrial switch patient (e and $\mathbf{f}$ ). Views $\mathbf{a}, \mathbf{c}$, and $\mathbf{e}$ show a four-chamber view and views b, $\mathbf{d}$, and $\mathbf{f}$ show an orthogonal plane passing through the apex and systemic outflow tract. The illustrated blood volumes are restricted to the plane of interest and are classified as direct flow (green), delayed ejection (blue), retained inflow (yellow), and residual volume (red) 

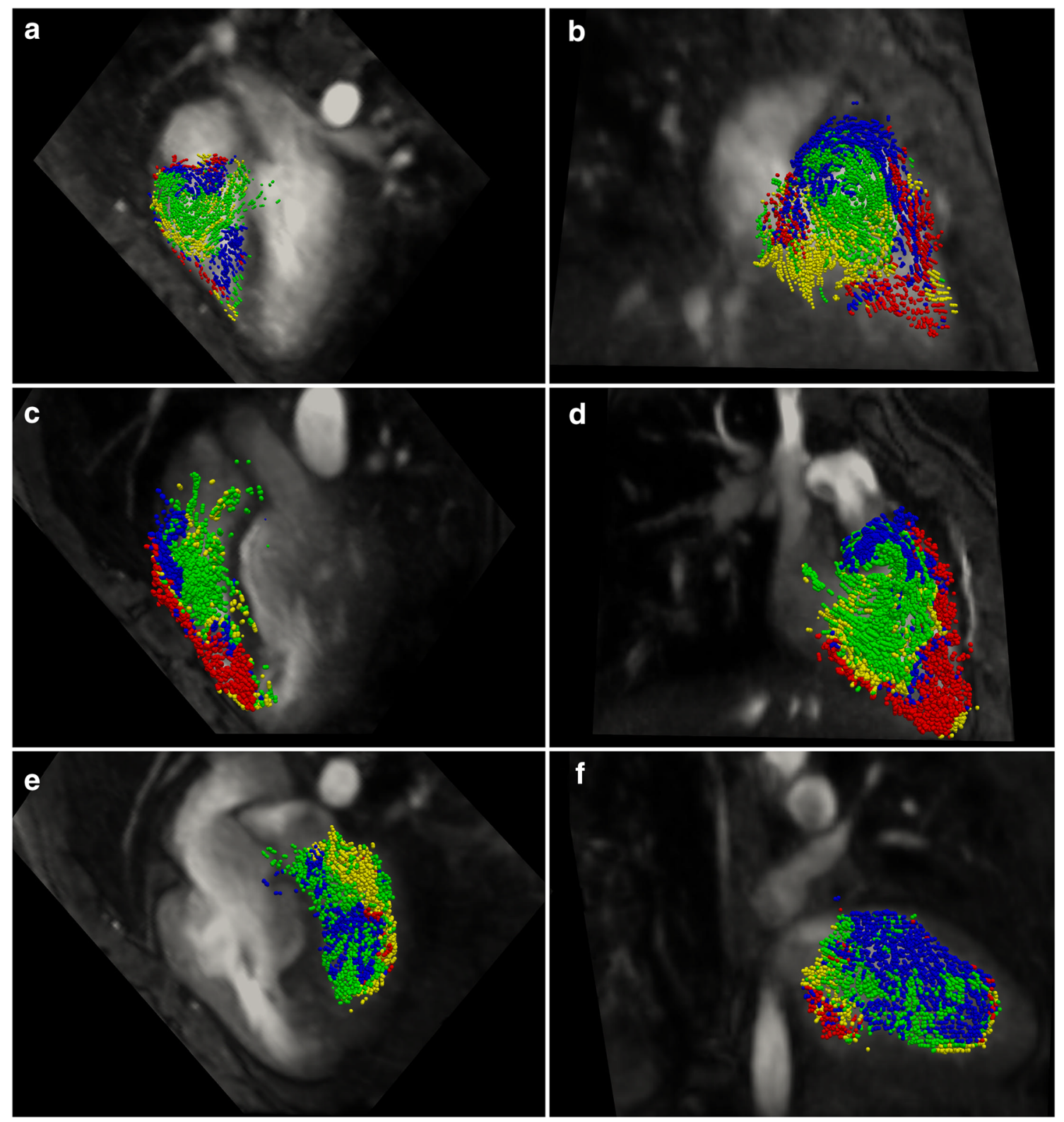

Fig. 3 The location of non-systemic ventricular blood volumes at the time point of end-diastole on two orthogonal views in a representative normal (a and b), d-TGA arterial switch (c and d), and a d-TGA atrial switch patient (e and f). Views a, c, and e show a four-chamber view and

subjects. There was a higher degree of mixing between the components (Figs. 2e, f and 3e, f). While Figs. 2 and 3 demonstrate a temporal snapshot of the flow order and disorder in representative subjects, Supplementary Movie 1 provides clear animations of the full dynamics for three illustrative subjects.

\section{Quantification of ventricle flow component volumes}

The fractions of systemic ventricular flow passing as direct flow and as delayed ejection were significantly lower in atrial switch patients compared with those in both arterial views $\mathbf{b}, \mathbf{d}$, and $\mathbf{f}$ show an orthogonal plane passing through the apex and outflow tract. The illustrated blood volumes are restricted to the plane of interest and are classified as direct flow (green), delayed ejection (blue), retained inflow (yellow), and residual volume (red)

switch patients ( $p<0.0001$ and $p<0.001$ respectively) and normal subjects ( $p=0.003$ and $p<0.001$ respectively). This was compensated by a significantly larger proportion of residual volume in atrial switch patients compared to arterial switch patients and normal subjects $(p<$ 0.0001 for both). Retained inflow fractions were not different. There were no differences between any flow fractions in arterial switch patients compared to normal subjects (Fig. 4a).

In the non-systemic ventricle, the retained inflow fraction in atrial switch patients was significantly higher than that in normal subjects $(p=0.02)$ and arterial switch patients $(p<$ 
0.001). Residual volume fractions were lower in atrial switch subjects compared to those in normal subjects $(p=0.02)$. The direct flow portion was significantly higher in arterial switch patients compared to both normal subjects $(p=0.007)$ and atrial switch patients $(p=0.01)$. Delayed ejection fractions were significantly lower in arterial switch patients compared to those in both normal subjects $(p<0.001)$ and atrial switch patients $(p<0.001)$ (Fig. 4b).

\section{Viscous energy losses}

Viscous energy losses showed large variability within all three subject groups (Table 2). EL $\mathrm{EL}_{v}$ and $\overline{E L_{v}}$ were significantly higher in the non-systemic ventricles of atrial switch subjects, compared to normal subjects ( $p=0.03$ for both). $\overline{E L_{v}}$ was significantly decreased in the systemic ventricle of atrial switch patients compared to that of both normal and arterial switch subjects ( $p=0.01$ and 0.005 respectively). There were no significant differences of $\mathrm{EL}_{v}$ or $\overline{E L_{v}}$ between normal subjects and arterial switch patients. It was visually observed that subjects with a high $\mathrm{EL}_{v}$ displayed greater disorder of the ventricle flow classifications. Additionally, regions of high rates of $\mathrm{EL}_{v}$ tended to correspond to locations of high mixing between separate flow components (Fig. 5).

\section{Discussion}

This study investigated the intraventricular flow volumes and dynamics in d-TGA patients after surgical repair of either atrial switch operation (Senning procedure) or arterial switch operation, in comparison with patients having normal cardiovascular anatomy. We assessed ventricle volumetrics (EDV, $\mathrm{SV}$, and EF) derived from cine short-axis stack MRI data and intraventricular flow classification using 4D-flow MRI. The intraventricular flow classification highlighted well-organised flow patterns in normal paediatric subjects, while varying levels of disorder were found in the arterial switch subjects and high disorder was observed in the flow of adult atrial switch subjects. Arterial switch patients showed a systemic ventricle behaving similarly to normal subjects and an increased proportion of non-systemic ventricle direct flow fraction. Visually, mixing of ventricular flow components corresponded to areas of high rates of viscous energy loss, which was elevated in atrial switch subjects.

Ventricle volumetrics showed expected results concordant with other studies. Normal subjects had EDV, SV, and EF measurements in agreement with published data for children [21]. Arterial switch subjects displayed larger systemic EDV and non-systemic EF values compared to normal subjects in our study; however, these numbers were within normal limits for children [21]. The atrial switch patients in our study had
Fig. 4 Pie chart representation of ventricular flow components of the systemic ventricle in d-TGA patients (atrial and arterial switch corrections) and normal subjects (upper panel) and the nonsystemic ventricle (lower panel)
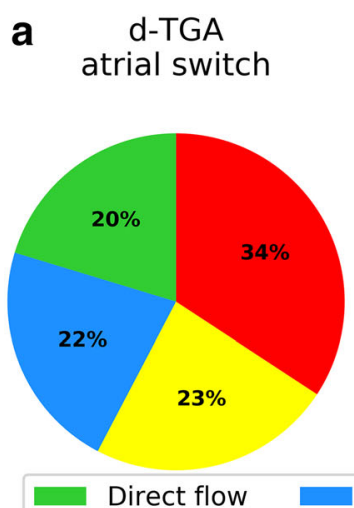

Direct flow
d-TGA arterial switch

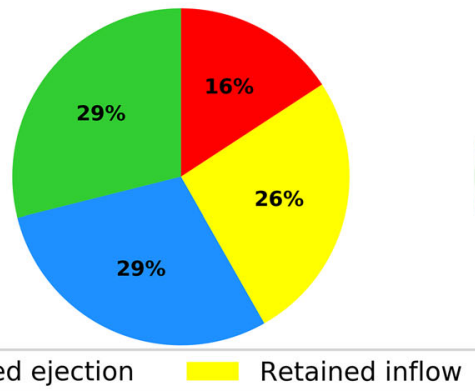

d-TGA arterial switch

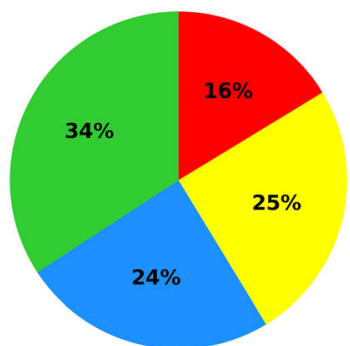

Retained inflow

\section{Normal}

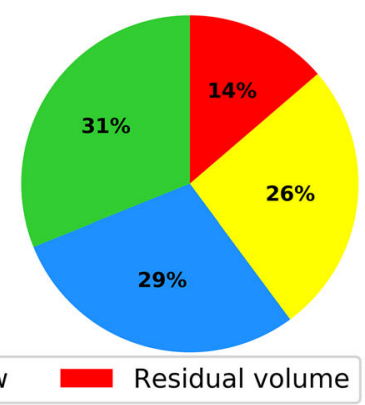

Normal

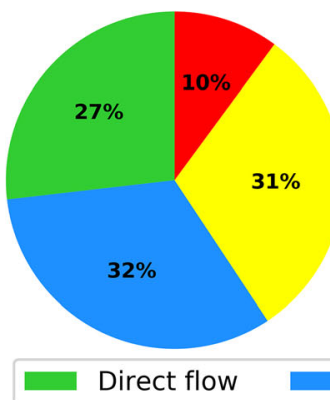

Delayed ejection

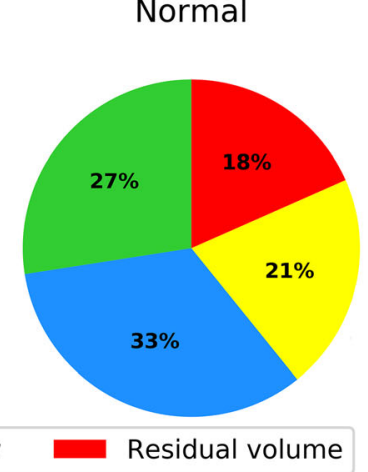


Table 2 Intraventricular viscous energy losses in d-TGA subjects following atrial or arterial switch operations and normal subjects

\begin{tabular}{|c|c|c|c|c|c|c|}
\hline & Atrial switch & Arterial switch & Normal & $\begin{array}{l}\text { Atrial vs } \\
\text { jarterial switch }\end{array}$ & $\begin{array}{l}\text { Normal vs } \\
\text { atrial switch }\end{array}$ & $\begin{array}{l}\text { Normal vs } \\
\text { arterial switch }\end{array}$ \\
\hline \multicolumn{7}{|c|}{ Viscous energy loss- $-\mathrm{EL}_{v}[\mathrm{~mJ}]$} \\
\hline Systemic ventricle & $0.11 \pm 0.07$ & $0.12 \pm 0.07$ & $0.08 \pm 0.05$ & 0.62 & 0.30 & 0.11 \\
\hline Non-systemic ventricle & $0.13 \pm 0.09$ & $0.09 \pm 0.06$ & $0.06 \pm 0.04$ & 0.17 & 0.03 & 0.21 \\
\hline \multicolumn{7}{|c|}{ Normalised viscous energy loss $-E L_{v}\left[\mathrm{~mJ} / \mathrm{m}^{3}\right]$} \\
\hline Systemic ventricle & $527 \pm 232$ & $993 \pm 560$ & $778 \pm 240$ & 0.005 & 0.01 & 0.22 \\
\hline Non-systemic ventricle & $900 \pm 493$ & $724 \pm 403$ & $547 \pm 194$ & 0.28 & 0.03 & 0.17 \\
\hline
\end{tabular}
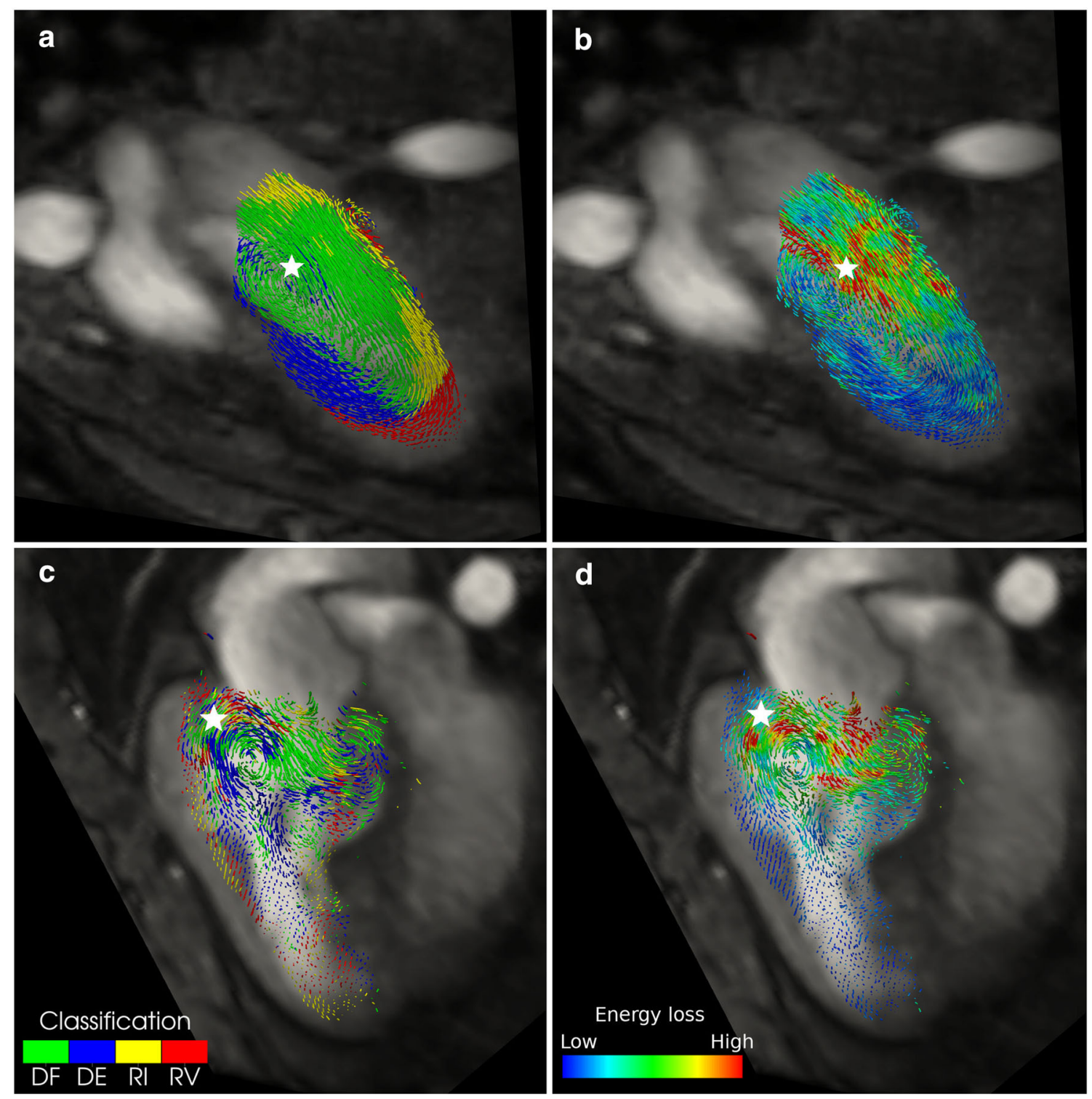

Fig. 5 Example of correlation between mixing between flow components and high rates of viscous energy loss in an illustrative normal subject (top row) and a d-TGA patient following atrial switch repair (bottom row). All panels show a snapshot of pathlines upon a plane of the systemic ventricle at approximately end-diastole. Panels a and $\mathbf{c}$ show the pathlines coloured by their respective classification as direct flow (DF), delayed ejection

(DE), retained inflow (RI), and residual volume (RV). Panels b and $\mathbf{d}$ show the same image, but with pathlines coloured by the relative magnitude of the rate of viscous energy loss. White stars in each panel indicate locations of mixing of DF and DE components and corresponding high rates of viscous energy loss 
significantly larger systemic EDV volumes and smaller EF compared with both normal and arterial switch subjects. These values were in agreement with previously published data [22]. In the non-systemic ventricle, atrial switch patients had no difference in EDV and SV compared with normal and arterial switch subjects and slightly larger EF compared to normal subjects, also in agreement with previously published results [23].

All normal subjects showed well-structured flow through the ventricles, matching that described in previous studies [17, 18, 24-26]. Atrial switch patients showed a significant decrease in direct flow fraction in the systemic ventricle. This is the morphological right ventricle for these subjects, many of whom had high end-diastolic volumes and low ejection fractions. Thus the reduced direct flow fractions are expected in this scenario. Decreased direct flow and increased residual volume portions have previously been associated with increased EDV and reduced EF values [27]. In the nonsystemic ventricle, flow portions of atrial switch patients were more similar to those of the systemic ventricle of normal subjects illustrating that the morphological left ventricle continues to behave representative of a systemic ventricle in atrial switch subjects. Both the transposed ventricles and disturbed inflows, due to redirection via baffles within the atria likely contribute to the differences observed. Arterial switch patients showed elevated direct flow fractions and a decreased delayed ejection fraction compared to normal subjects in the non-systemic ventricle. This may be a result of the slightly different geometry of the outflow tract and orientation of the main pulmonary artery, which is a common outcome following surgery for these subjects [28].

We observed clear flow disorder in some arterial switch and most atrial switch d-TGA subjects, illustrated by mixing of the ventricular flow components, in distinct contrast to the ordered flow patterns observed in all normal, paediatric, subjects and represented in previous publications including healthy adult subjects $[18,25]$. Local areas of mixing, especially between flow components that were separate at endsystole, such as the direct flow and delayed ejection portions, corresponded to higher rates of $\mathrm{EL}_{v}$. Note that the high enddiastolic volumes of the systemic ventricles of atrial switch subjects resulted in low normalised viscous energy losses where high absolute viscous energy losses were observed. Our results are in agreement with other studies that have reported elevated viscous energy loss in disrupted flow dynamics $[20,29-31]$. The intraventricular dynamics observed in normal subjects were highly structured and matched those previously described $[17,18,24,26]$. The inflow volume replaces the delayed/residual volume and drives these volumes towards the outflow tract in preparation for ejection during the following cycle. In the systemic ventricles of subjects showing flow disturbance and mixing of components, the most obvious difference was a disruption of the inlet to apex to the outlet flow path. As a result, flow particles inevitably move "against the flow" and induce friction, which is quantified as viscous energy loss. Elevated viscous energy losses in the presence of disturbance of normal intraventricular flow patterns, specifically, altered vortex ring formation, which may be analogous to the disorganised ventricular flow patterns have been previously described [30].

Other studies have investigated kinetic energy levels in congenital heart disease cohorts, especially patients after Tetralogy of Fallot repair [12-14]. We choose not to pursue this route of investigation preferring to focus on a marker of absolute inefficiency represented by viscous energy loss. This is an irreversible conversion of kinetic to thermal energy due to viscous interactions and has shown promising results in investigations involving disturbed flow dynamics $[15,20$, 32, 33]. In patients after Fontan palliation, for example, viscous energy loss was significantly elevated compared to controls, predominantly in patients with discordant inflow versus outflow underlying the impact of structured flow paths and ventricular performance [15].

\section{Limitations}

This study examined three distinct groups: normal anatomy subjects and subjects with d-TGA corrected by arterial or atrial switch repair. The large age difference between the atrial switch group and the other two groups, as well as the significantly different anatomy and relatively low subject numbers limit the impact of inter-group comparisons. The normal anatomy and arterial switch group covered age ranges representing high growth and development [21]. This may explain the disagreement with previously published ventricle flow classification proportions [34]. A recent study covering a similar age range as the current study gives similar left ventricle flow proportions for normal subjects [27], supporting our results and suggesting an age-related change in ventricular haemodynamics.

Some limitations in the accuracy of the techniques applied are to be expected. Using a free-breathing protocol without respiratory gating is a necessity of our clinical practice and this may result in inaccuracies for pathline tracking; however, quality control comparisons of pathline-based stroke volumes compared with volumetric-based stroke volumes suggest this was not critical.

The observations made with regard to the degree of flow component mixing as well as the correlation of this with viscous energy losses are qualitative ones and have not been made under a rigorous verification or quantification process. These results deserve further exploration in a dedicated study, though it is acknowledged that repeatable quantification of such dynamics is difficult.

This study involves an advanced analysis of 4D-flow data, with high user input and long processing time, which is not readily available at a clinical level. The measurement of 
viscous energy loss is a parameter that relies on the derivative of the velocity field and thus is subject to amplification of errors that may be present due to noise. A typical technique for reducing noise by minimising the encoding velocity was not adopted in this study [35]. Additionally, viscous energy loss measures are dependent on spatial resolution and are known to be underestimated in areas of turbulence, which may be present in the mixing zones observed [36].

\section{Conclusions}

4D-flow MRI identifies both quantitative and qualitative differences in ventricular flow patterns in arterial and atrial switch d-TGA patients and normal subjects. Arterial switch patients generally show flow behaviour and quantification similar to normal subjects. Atrial switch patients displayed disordered ventricular flow compared with the younger cohort of normal subjects in our study and with previously published normal data. Flow disorder in the ventricle leads to increased viscous energy losses.

Supplementary Information The online version contains supplementary material available at https://doi.org/10.1007/s00330-021-07813-0.

Funding Open Access funding provided by Universität Zürich.

\section{Declarations}

Guarantor The scientific guarantor of this publication is FMC.

Conflict of interest The authors of this manuscript declare no relationships with any companies whose products or services may be related to the subject matter of the article.

Statistics and biometry No complex statistical methods were necessary for this paper.

Informed consent Written informed consent was obtained from all subjects (patients) (or parents/guardians if applicable) in this study.

Ethical approval Institutional Review Board approval was obtained.

\author{
Methodology \\ - retrospective \\ - observational \\ - performed at one institution
}

Open Access This article is licensed under a Creative Commons Attribution 4.0 International License, which permits use, sharing, adaptation, distribution and reproduction in any medium or format, as long as you give appropriate credit to the original author(s) and the source, provide a link to the Creative Commons licence, and indicate if changes were made. The images or other third party material in this article are included in the article's Creative Commons licence, unless indicated otherwise in a credit line to the material. If material is not included in the article's Creative Commons licence and your intended use is not permitted by statutory regulation or exceeds the permitted use, you will need to obtain permission directly from the copyright holder. To view a copy of this licence, visit http://creativecommons.org/licenses/by/4.0/.

\section{References}

1. Villafañe J, Lantin-Hermoso MR, Bhatt AB et al (2014) DTransposition of the great arteries. J Am Coll Cardiol 64:498 511. https://doi.org/10.1016/j.jacc.2014.06.1150

2. Kiener A, Kelleman M, McCracken C et al (2018) Long-term survival after arterial versus atrial switch in d-transposition of the great arteries. Ann Thorac Surg 106:1827-1833. https://doi.org/10.1016/ j.athoracsur.2018.06.084

3. Dennis M, Kotchetkova I, Cordina R, Celermajer DS (2018) Longterm follow-up of adults following the atrial switch operation for transposition of the great arteries - a contemporary cohort. Hear Lung Circ 27:1011-1017. https://doi.org/10.1016/j.hlc.2017.10. 008

4. Venkatesh P, Evans AT, Maw AM et al (2019) Predictors of late mortality in D-transposition of the great arteries after atrial switch repair: systematic review and meta-analysis. J Am Heart Assoc 8. https://doi.org/10.1161/JAHA.119.012932

5. Kempny A, Wustmann K, Borgia F et al (2013) Outcome in adult patients after arterial switch operation for transposition of the great arteries. Int J Cardiol 167:2588-2593. https://doi.org/10.1016/j. ijcard.2012.06.066

6. Moe TG, Bardo DME (2018) Long-term outcomes of the arterial switch operation for D-transposition of the great arteries. Prog Cardiovasc Dis 61:360-364. https://doi.org/10.1016/j. pcad.2018.08.007

7. Losay J, Touchot A, Serraf A et al (2001) Late outcome after arterial switch operation for transposition of the great arteries. Circulation 104:I121-I126. https://doi.org/10.1161/hc37t1.094716

8. Fricke TA, Konstantinov IE (2019) Arterial switch operation: operative approach and outcomes. Ann Thorac Surg 107:302-310. https://doi.org/10.1016/j.athoracsur.2018.06.002

9. Markl M, Frydrychowicz A, Kozerke S et al (2012) 4D flow MRI. J Magn Reson Imaging 36:1015-1036. https://doi.org/ 10.1002/jmri.23632

10. Dyverfeldt P, Bissell M, Barker AJ et al (2015) 4D flow cardiovascular magnetic resonance consensus statement. J Cardiovasc Magn Reson 17:72. https://doi.org/10.1186/s12968-015-0174-5

11. Rizk J (2020) 4D flow MRI applications in congenital heart disease. Eur Radiol. https://doi.org/10.1007/s00330-020-07210-z

12. Jeong D, Anagnostopoulos PV, Roldan-Alzate A et al (2015) Ventricular kinetic energy may provide a novel noninvasive way to assess ventricular performance in patients with repaired tetralogy of Fallot. J Thorac Cardiovasc Surg 149:1339-1347. https://doi. org/10.1016/j.jtcvs.2014.11.085

13. Sjöberg P, Bidhult S, Bock J et al (2018) Disturbed left and right ventricular kinetic energy in patients with repaired tetralogy of Fallot: pathophysiological insights using 4D-flow MRI. Eur Radiol 28:1-11. https://doi.org/10.1007/s00330-018-5385-3

14. Fredriksson A, Trzebiatowska-Krzynska A, Dyverfeldt $\mathrm{P}$ et al (2018) Turbulent kinetic energy in the right ventricle: potential MR marker for risk stratification of adults with repaired Tetralogy of Fallot. J Magn Reson Imaging 47:1043-1053. https://doi.org/10. 1002/jmri.25830

15. Kamphuis VP, Elbaz MSM, Van Den Boogaard PJ et al (2019) Disproportionate intraventricular viscous energy loss in Fontan 
patients: analysis by 4D flow MRI. Eur Heart J Cardiovasc Imaging 20:323-333. https://doi.org/10.1093/ehjci/jey096

16. Eriksson J, Carlhäll CJ, Dyverfeldt P et al (2010) Semi-automatic quantification of 4D left ventricular blood flow. J Cardiovasc Magn Reson 12:9. https://doi.org/10.1186/1532-429X-12-9

17. Eriksson J, Dyverfeldt P, Engvall J et al (2011) Quantification of presystolic blood flow organization and energetics in the human left ventricle. Am J Physiol Heart Circ Physiol 300:H2135-H2141. https://doi.org/10.1152/ajpheart.00993.2010

18. Fredriksson AG, Zajac J, Eriksson J et al (2011) 4-D blood flow in the human right ventricle. Am J Physiol Circ Physiol 301:H2344 H2350. https://doi.org/10.1152/ajpheart.00622.2011

19. Lai P, Shimakawa A, Cheng JY et al (2015) Sub-8-minute cardiac four dimensional flow MRI using kat ARC and variable density signal averaging. J Cardiovasc Magn Reson 17:Q36. https://doi. org/10.1186/1532-429X-17-S1-Q36

20. Barker AJ, van Ooij P, Bandi K et al (2014) Viscous energy loss in the presence of abnormal aortic flow. Magn Reson Med 72:620 628. https://doi.org/10.1002/mrm.24962

21. Kawel-Boehm N, Maceira A, Valsangiacomo-Buechel ER et al (2015) Normal values for cardiovascular magnetic resonance in adults and children. J Cardiovasc Magn Reson 17:29. https://doi. org/10.1186/s12968-015-0111-7

22. Fratz S, Hager A, Busch R et al (2008) Patients after atrial switch operation for transposition of the great arteries can not increase stroke volume under dobutamine stress as opposed to patients with congenitally corrected transposition. Circ J 72:1130-1135. https:// doi.org/10.1253/circj. 72.1130

23. Pettersen E, Lindberg H, Smith HJ et al (2008) Left ventricular function in patients with transposition of the great arteries operated with atrial switch. Pediatr Cardiol 29:597-603. https://doi.org/10. 1007/s00246-007-9156-1

24. Stoll VM, Hess AT, Rodgers CT et al (2019) Left ventricular flow analysis novel imaging biomarkers and predictors of exercise capacity in heart failure. Circ Cardiovasc Imaging 12:1-12. https:// doi.org/10.1161/CIRCIMAGING.118.008130

25. Bolger A, Heiberg E, Karlsson M et al (2007) Transit of blood flow through the human left ventricle mapped by cardiovascular magnetic resonance. J Cardiovasc Magn Reson 9:741-747. https://doi. org/10.1080/10976640701544530

26. Svalbring E, Fredriksson A, Eriksson J et al (2016) Altered diastolic flow patterns and kinetic energy in subtle left ventricular remodeling and dysfunction detected by 4D flow MRI. PLoS One 11:1-12. https://doi.org/10.1371/journal.pone.0161391

27. Schäfer M, Browne LP, Jaggers J et al (2019) Abnormal left ventricular flow organization following repair of tetralogy of
Fallot. J Thorac Cardiovasc Surg. https://doi.org/10.1016/j. jtcvs.2019.11.010

28. Geiger J, Hirtler D, Bürk J et al (2014) Postoperative pulmonary and aortic 3D haemodynamics in patients after repair of transposition of the great arteries. Eur Radiol 24:200-208. https://doi.org/10. 1007/s00330-013-2998-4

29. Han QJ, Witschey WRT, Fang-Yen CM et al (2015) Altered right ventricular kinetic energy work density and viscous energy dissipation in patients with pulmonary arterial hypertension: a pilot study using 4D flow MRI. PLoS One 10:e0138365. https://doi.org/10. 1371/journal.pone. 0138365

30. Elbaz MSM, van der Geest RJ, Calkoen EE et al (2017) Assessment of viscous energy loss and the association with three-dimensional vortex ring formation in left ventricular inflow: In vivo evaluation using four-dimensional flow MRI. Magn Reson Med 77:794-805. https://doi.org/10.1002/mrm.26129

31. Stugaard M, Koriyama H, Katsuki K et al (2015) Energy loss in the left ventricle obtained by vector Flow mapping as a new quantitative measure of severity of aortic regurgitation: a combined experimental and clinical study. Eur Heart J Cardiovasc Imaging 16:723730. https://doi.org/10.1093/ehjci/jev035

32. Elbaz MSM, Calkoen EE, Westenberg JJM et al (2014) Vortex flow during early and late left ventricular filling in normal subjects: quantitative characterization using retrospectively-gated $4 \mathrm{D}$ flow cardiovascular magnetic resonance and three-dimensional vortex core analysis. J Cardiovasc Magn Reson 16:78. https://doi.org/10. 1186/s12968-014-0078-9

33. Shibata M, Itatani K, Hayashi T et al (2018) Flow energy loss as a predictive parameter for right ventricular deterioration caused by pulmonary regurgitation after Tetralogy of Fallot repair. Pediatr Cardiol 39:731-742. https://doi.org/10.1007/s00246-018-1813-Z

34. Stoll VM, Loudon M, Eriksson J et al (2018) Test-retest variability of left ventricular 4D flow cardiovascular magnetic resonance measurements in healthy subjects. J Cardiovasc Magn Reson 20:1-10. https://doi.org/10.1186/s12968-018-0432-4

35. Callaghan FM, Kozor R, Sherrah AG et al (2016) Use of multivelocity encoding 4D flow MRI to improve quantification of flow patterns in the aorta. J Magn Reson Imaging 43:352-363. https:// doi.org/10.1002/jmri.24991

36. Casas B, Lantz J, DyverfeldT P, Ebbers T (2016) 4D Flow MRIbased pressure loss estimation in stenotic flows: evaluation using numerical simulations. Magn Reson Med 75:1808-1821. https:// doi.org/10.1002/mrm. 25772

Publisher's note Springer Nature remains neutral with regard to jurisdictional claims in published maps and institutional affiliations. 